\title{
Energy Management Strategies based on Efficiency Map for Fuel Cell Hybrid Vehicles
}

\author{
Diego Feroldi (corresponding author), Maria Serra, and Jordi Riera \\ Institut de Robòtica i Informàtica Industrial (CSIC-UPC) \\ C. Llorens i Artigas 4, 08028 Barcelona, Spain \\ E-mail: $\underline{\text { dferoldi@,iri.upc.edu }}$
}

Telephone: 3493 4015754; fax: 34934015750

\begin{abstract}
The addition of a fast auxiliary power source like a supercapacitor bank in fuel cellbased vehicles has a great potential because permits a significant reduction of the hydrogen consumption and an improvement of the vehicle efficiency. The Energy Management Strategies, commanding the power split between the power sources in the hybrid arrangement to fulfil the power requirement, perform a fundamental role to achieve this objective. In this work, three strategies based on the knowledge of the fuel cell efficiency map are proposed. These strategies are attractive due to the relative simplicity of the real time implementation and the good performance. The strategies are tested both in a simulation environment and in an experimental setup using a $1.2-\mathrm{kW}$ PEM fuel cell. The results, in terms of hydrogen consumption, are compared with an optimal case, which is assessed trough an advantageous technique also introduced in this work and with a pure fuel cell vehicle as well. This comparative reveals high efficiency and good performance, allowing to save up to $26 \%$ of hydrogen in urban scenarios.
\end{abstract}

Keywords: Proton exchange membrane; Fuel Cell Hybrid Vehicle; Automotive application; Energy Management Strategy; Supercapacitor; Efficiency map 


\section{Introduction}

Given the serious environmental problems and the anticipated fuel shortage for the next decades it is important to find more efficient forms of using the power resources, affecting minimally the environment. According to different sources, such as [1], global oil reserves are only sufficient for around 40 years with the current level of oil production. However, the global economy is rapidly increasing with a subsequent increase in oil consumption: the total primary energy consumption has grown from 6128 Mtoe in 1973 to 11435 Mtoe in 2005; meanwhile, the $\mathrm{CO}_{2}$ emission has grown from $15661 \mathrm{Mt}$ to $7136 \mathrm{Mt}$ in the same period [2]. Thus, oil reserves will therefore be probably exhausted in a much shorter time period.

The main advantage of fuel cell-based vehicles as opposed to those with internal combustion engine is that in the former the energy conversion is direct (without combustion), allowing a higher efficiency and practically null emission of polluting agents. The efficiency of a fuel cell-based vehicle using hydrogen is twice the efficiency of a gasoline vehicle [3-4]. Moreover, unlike the internal combustion engines, the fuel cell efficiency is also high at partial loads: a vehicle running in urban or suburban areas is demanding a small fraction of the rated fuel cell power most of the time [5]. Thus, fuel cell-based vehicles have long term potential to be the mainstream vehicle in the future because they have almost zero emission and compatible driving range to internal combustion vehicles [6].

Fuel Cell Hybrid Vehicles (FCHV) is a promising fuel cell application that has taken more and more importance in the last years and is considered the most attractive longterm option for passenger cars [7]. Hybridization in fuel cell-based vehicles consists in 
adding a supplementary energy storage element (e.g., a battery or a supercapacitor bank) to the primary power source (i.e., the fuel cell system) in order to adequate in an optimum way the energy generation to the consumption. This procedure has important advantages, allowing a greater reduction of the hydrogen consumption. Depending on how the necessary power is obtained, a minimization of the hydrogen consumption can be achieved: the Energy Management Strategies (EMSs) are algorithms which determine at each sampling time the power generation split between the Fuel Cell System (FCS) and the Energy Storage System (ESS) in order to fulfil the power balance between the load power and the power source.

The EMSs in FCHV is an open issue, existing different approaches in the literature. In the work of Thounthong et al. [8], the strategy is based on DC link voltage regulation though the control of the power converters but does not deal with the system efficiency. Jung et al. [9] propose a control strategy with two objectives, to obtain high efficiency and to maintain a minimum state of charge in the batteries, giving priority to the second. On the other hand, other literature works are focused on strategies based on optimization theory. Paganelli et al. [10] present a method based on a control strategy called Equivalent Consumption Minimization Strategy. This strategy demonstrates to be robust under a wide range of operating conditions. Nevertheless, the efficiency is sensible to several parameters. In the same line, Rodatz et al. [11] implement a real time control by using the same concept of equivalent hydrogen consumption, showing experimental results. Another optimization algorithm, presented in [12], diminishes a cost function depending only on system variables considered at the present time but the conclusion is that the efficiency is not significantly improved. 
With regard to the energy storage element in FCHV, some publications in the literature analyze the feasibility and performance of supercapacitors. Karden et al. [13], analyzing energy storage devices for hybrid electric vehicles, conclude that supercapacitors are effective for pulse power applications. Kotz et al. [14] analyze a pilot system on small scale that represents a hybrid vehicle with fuel cell (FC) and supercapacitors, demonstrating that supercapacitors are excellent energy storage devices, which can be efficiently used in vehicle applications for energy braking recovering and peak power reinforcing. Another approach using supercapacitors is found in the work of Caux et al. $[15,16]$ in a tramway application. Schiffer et al. [17] and Kim et al. [18] also show the benefits of using a fuel cell-supercapacitor system for FCHVs.

The main objective of EMSs is to improve the efficiency reducing the hydrogen consumption along the driving cycle. To find a global optimal solution, control techniques where a minimization problem is resolved have been studied, e.g. in [19]. In general, these techniques do not offer a causal solution, and, in consequence, are not feasible because it is assumed that the future driving cycle is entirely known a priori. Nevertheless, their results can be used to make comparative analysis of the performance of new strategies in study. On the other hand, strategies which deal with local optimization are convenient for real implementation. Another approach, particularly convenient to work in real time operation, is the type of strategies where the system operation is based on heuristic rules derived from the knowledge of the performance of the system's components. These strategies can achieve a comparable performance to those strategies where some optimization technique is utilized. 
In this work, three energy management strategies for FCHVs are presented. The strategies are based on the knowledge of the fuel cell efficiency map: two of them are heuristic type strategies and the third strategy is based on constrained nonlinear programming. Besides, a novel method to asses the optimal consumption knowing the future driving profile is presented. This last result is used to compare the performance of the proposed strategies. The FCHV in study is based on a small car with the energy storage system composed by a supercapacitor bank and the entire system is modelled using ADVISOR (ADvanced VehIcle SimulatOR is a toolbox developed by the National Renewable Energy Laboratory with the aim of analyzing the performance and fuel economy of conventional, electric, and hybrid vehicles $[20,21]$ ) in MATLAB/SIMULINK. The proposed strategies are tested both in simulation and in an experimental setup, showing an auspicious performance.

The organization of the paper is as follows. In Section 2, Fuel Cell Hybrid Vehicles are introduced showing the advantages and the mechanism that allows improving the efficiency. Then, the proposed Energy Management Strategies are introduced in Section 3. In Section 4, the simulation results obtained using these strategies are presented, including the description of the procedure to asses the minimum consumption. In

Section 5, the experimental validation is presented, describing the experimental setup and showing the comparative results. Finally, the discussion of the results and the conclusions of the work are in Section 6 and 7, respectively.

\section{Fuel Cell Hybrid Vehicles (FCHVs)}

In a Fuel Cell Hybrid Vehicle (FCHV), the powertrain is composed by the fuel cell system and an Energy Storage System (ESS) in order to increase the efficiency of the 
integrated system (Fig. 1). Given a certain load power $P_{\text {load }}(t)$, this can be supplied with some power from the fuel cell system, $P_{f c s}(t)$, being the rest of power supplied by the energy storage system, $P_{\text {ess }}(t)$. The power balance in the DC bus must be fulfilled at every time:

$P_{f c s}(t) \eta_{B}+P_{e s s}(t) \eta_{B / B} \eta_{\text {ess }}=P_{\text {load }}(t) \quad \forall t$,

where $\eta_{B}, \eta_{B / B}$, and $\eta_{\text {ess }}$ are the efficiency of the power converter that connects the fuel cell with the bus, the efficiency of the power converter that connects the ESS with the bus, and the efficiency of the ESS, respectively. The control of the DC/DC converters is beyond the scope of this paper. We assume that the converters are controlled and their efficiencies are known.

In a $\mathrm{FCHV}$, three different mechanisms allow this efficiency improvement:

1. In automotive applications, it is possible to recover energy from regenerative braking, allowing a considerable improvement in the hydrogen economy. The recovered energy from regenerative braking may represent an important fraction of the economy improvement. However, the amount of energy that is possible to recover is strongly dependent on the driving profile. In urban driving cycles, in which there is considerable braking, the percentage of regenerated energy is much greater than in non-urban cycles where this percentage is quite small.

2. The efficiency of a FCS is high at partial loads. Therefore, it is convenient to perform an energy management in which the fuel cell is preferably operated in its maximum efficiency zone. Thus, taking advantage of an auxiliary power source, e.g. the storage system, and an adequate energy strategy, it is possible to maintain the fuel cell 
operating at its maximum efficiency region most of the time and reduce the hydrogen consumption.

3. In a pure fuel cell vehicle, the fuel cell must be sized to meet the largest load. One important advantage of hybridization is that it allows a diminution of the rated power of the fuel cell stack in these types of applications. Therefore, it is possible to meet the demand with a smaller FCS in combination with an energy storage system. Besides, downsizing the FCS has an impact on the hydrogen economy in three ways: $i$ ) downsizing leads to improvements in the hydrogen economy when the peak efficiency of the FC stack is shifted to better match with the power requirements for a given load cycle, ii) downsizing leads to a smaller compressor reducing the parasitic losses, and iii) downsizing reduces the total mass of the vehicle.

Although the main motivation for introducing hybridization in fuel cell systems is to improve the hydrogen economy, the benefits are not limited to that. In fact, hybridization is also useful to solve two important problems in fuel cell control:

1. The dynamics of FCS are relatively slow, mainly because of the dynamics of the air compressor and the manifold-filling dynamics [22]. In this context, an energy flow from an energy storage device with high specific power output is useful to improve the transient response. In the literature, this issue is not completely solved. In [23], it is concluded that it is feasible to use a FCS in a load-following mode without any storage device. In that work, the conclusions are based on results obtained from experimental data working with a scaled-down FCS through the Federal Urban Driving Schedule (FUDS). It is remarked that it is possible to pull short bursts of power from the stack, due to the charge in the double layer (CDL) capacitance. However, several publications, 
for example [14], [24] or [4], conclude that the use of energy storage devices is beneficial from both the system dynamics and the hydrogen economy.

2. Hybridization can help avoiding oxygen starvation by means of the energy supply from the energy storage elements. Thus, the peaks of actual current demand to the fuel cell are lower than in the pure fuel cell case where no auxiliary power source is present. Oxygen Starvation is a complicated phenomenon that occurs when the oxygen partial pressure falls below a critical level at any point in the cathode. It can be produced by an abrupt increase in the current demand (that produces a rapid increase in the oxygen consumption due to the cathode electrochemical reaction) and an insufficient air supply. This phenomenon can be observed by a rapid decrease in the cell voltage and in severe cases can produce a short circuit and a hot spot on the surface of the membrane cell [25]. The phenomenon is not well understood and more information from electrochemical studies is needed to be able to quantify the critical level of oxygen concentration below which permanent damage is produced or, perhaps more probably, the combination of current level variation and its time duration.

\section{Energy Management Strategies for FCHVs}

In this work, a search for new EMSs in FCHVs is addressed, and, despite the Fuel Cell Hybrid Systems have other applications, we concentrate our attention in automotive applications. The objective is to improve the performance of a FC hybrid vehicle in terms of hydrogen economy and transient response.

In Fig. 1 is represented the proposed topology of the system, where the FCS is connected to the DC bus through a step-up power converter (Boost converter), whereas the ESS is connected to the DC through a bidirectional power converter (Buck-Boost 
converter) allowing the energy flow from regenerative braking. With regard to the load, which is an AC induction motor, it is fed through a DC-AC inverter. This topology is common in the three strategies proposed. In the same fig. 1, the energy flows in the FCHS are also represented: the regenerated energy from the load to the ESS bank, the charging energy from the FCS to the ESS, the boosting energy from the ESS to the load, and the fuel cell energy that directly supplies the load from FCS.

\subsection{Objectives of EMSs in FCHVs}

The hydrogen consumption map of a FCS clearly reveals a zone, below a limit power, where the hydrogen consumption is very high meanwhile the zone above this limit power has a significant lower consumption as can be seen in Fig. 2. The principal objective of the energy management strategy is to minimize a cost function $J$, a mathematical expression that represents the cumulative hydrogen consumption during the time:

$\min _{X} J(X)$ subject to $H(X)=0$ and $G(X) \leq 0$

where the vector $X$ is:

$$
X=\left[\begin{array}{c}
P_{f c s}(k) \\
P_{e s s}(k)
\end{array}\right]
$$

This means that the EMS has to determine the optimal value of the Fuel Cell System power, $P_{f c s}(t)$, and the Energy Storage System power, $P_{\text {ess }}(k)$, in order to minimize the cost function $J(X)$ :

$J(X)=\sum_{k=0}^{N_{c}} F(X(k)) \Delta T$ 
where $N_{c}=t_{c} / \Delta T$ is the duration of the driving cycle, assuming a constant sampling period $\Delta T=1 s$, and $F(X(k))$ is the hydrogen consumption according to the hydrogen consumption map of the FCS:

$$
F(X(k))=\operatorname{Cons}_{H 2}\left(P_{f c s}(k)\right)
$$

The constraint $H(X)=0$ in (2) means that at every time the power load in the DC bus must be satisfied by the power from the FC or the ESS:

$$
P_{f c s}(k) \eta_{B}+P_{e s s}(k) \eta_{B / B} \eta_{e s s}=P_{\text {load }}(k) \quad k=0,1, \ldots, N_{c}
$$

where the efficiencies $\eta_{B}, \eta_{B / B}$, and $\eta_{e s s}$ are assumed to be constants.

On the other hand, the constraint $G(X) \leq 0$ in (2) states the constraints in the FCS and the ESS power for all $k$ :

$$
\begin{aligned}
& P_{f c s, \text { min }} \leq P_{f c s}(k) \leq P_{f c s, \text { max }} \\
& \Delta P_{f c s, \text { fall rate }} \leq \frac{d P_{f c s}(k)}{d t} \leq \Delta P_{f c s, \text { rise rate }} \\
& P_{\text {ess,dischrg max }}(\operatorname{SoE}(k)) \leq P_{\text {ess }}(k) \leq P_{\text {ess, chrg max }}(\operatorname{SoE}(k)) \\
& S o E_{\text {min }} \leq \operatorname{SoE}(k) \leq S o E_{\max }
\end{aligned}
$$

The inequality (7) states the restrictions in the FCS power. The maximum power is limited by the FCS rated power, whereas the minimum FCS power command must be limited to a value below which it is not suitable to operate because the parasitic load is too large, reducing the system net power.

There is a phenomenon of delay between the onset of the load on the FCS and the response of the reactant supply system which may lead in a "starvation" of reactants in the FCS. This may be avoided by restricting the dynamics required by the load [12]. The same approach is followed in [26] and [14] where the maximum positive rise is 
limited to avoid damage in the stack. Therefore, in our work, the FCS power load is increased no faster than a certain power rise rate $\left(\Delta P_{f c \text {, rise rate }}\right)$. Besides, we propose to operate the FCS in such a way that the power is decreased no faster than a certain power fall rate $\left(\Delta P_{f c s, f a l l}\right.$ rate $)$ to prevent overpressure into the stack. Both situations can be managed by using the ESS as a power buffer. These restrictions are formulated in (8). With regard to the ESS, the maximum and minimum power flows are also limited, as is formulated in (9). The maximum power that the ESS can supply or store depends on the actual voltage in the ESS, $V_{\text {ess }}(k)$, the maximum voltage $V_{\text {ess, } \max }(k)$, and the minimum voltage $V_{\text {ess, } \min }(k)$ :

$$
\begin{aligned}
& P_{e s s, \text { chrg max }}=\frac{\left(V_{e s s}(k)-V_{e s s, \max }\right) V_{e s s}(k)}{R_{d}} \text { during charging } \\
& P_{\text {ess, disch max }}=\frac{\left(V_{\text {ess }}(k)-V_{\text {ess, min }}\right) V_{\text {ess }}(k)}{R_{d}} \text { during discharging }
\end{aligned}
$$

where $R_{d}$ is the ESS internal resistance.

The State of Energy in the ESS, $\operatorname{SoE}(k)$, is defined as:

$$
\operatorname{SoE}(k):=\frac{E_{\text {ess }}(k)}{E_{\text {cap }}} \times 100 \quad[\%]
$$

where $E_{c a p}$ is the maximum energy that the ESS is capable to store. Thus, it is possible to express the maximum $P_{\text {ess }}(k)$ that is possible to charge or discharge, as a function of $\operatorname{SoE}(k)$ :

$$
\begin{aligned}
& P_{\text {ess, chrg max }}(k)=k_{\text {ess }}\left(\operatorname{SoE}(k)-S o E_{\text {max }}\right) \\
& P_{\text {ess, dischrg max }}(k)=k_{\text {ess }}\left(\operatorname{SoE}(k)-S o E_{\text {min }}\right)
\end{aligned}
$$

whith: 
$k_{\text {ess }}=\frac{k E_{\text {cap }}}{R_{d} C_{R}}$

where $R_{d}$ and $C_{R}$ are the internal resistance and the capacitance of the supercapacitors, and $k$ is a constant depending in their particular technology. According to the sign convention employed, the power is negative when the ESS is in charging mode; meanwhile it is positive when the ESS is in discharging mode.

The internal resistance of supercapacitors is extremely low and the capacitance is exceptionally high, allowing a very fast operation both during charging and discharging. In the supercapacitors employed in this work, they are $R_{d}=0.019 \Omega$ and $C_{R}=58 \mathrm{~F}$, thus, $k_{\text {ess }}=479 \mathrm{~kW}$.

The temporal behaviour of FCS is fundamentally conditioned by the compressor dynamics and the filling dynamics. Besides de minimization of $J$, another important EMS objective is to overcome this drawback by means of an adequate energy management strategy. In that sense, the energy management strategy acts as follows: if the power requested to the FCS exceeds a determined maximum rise rate, then the power rise rate that the FCS actually gives is limited and the rest of power is supplied by the ESS if it is possible according to the present value of $\operatorname{SoE}(k)$. If, on the contrary, the power requested to the FCS exceeds the maximum fall rate, then the actual power fall rate is limited and the surplus power is absorbed by the ESS whenever there is sufficient storage capacity.

\subsection{Strategy based on the FCS efficiency map}

One of the most relevant characteristic of a FCS is the efficiency map (Fig. 2), a chart that shows how the efficiency changes with the load power. It is supposed that the FCS 
operating point is controlled [27] and, thus, external parameters such the ambient temperature have no influence in the efficiency map. The first strategy proposed in this work is a quasi-load-following strategy where the FCS is operated in an advantageous zone where the efficiency is high. In this case, the operating zone is constrained between an inferior limit $\left(P_{f c s, l o}\right)$ and a superior limit $\left(P_{f c s, h i}\right)$. The superior limit is imposed by the maximum power that the fuel cell can deliver (i.e., $P_{f c s, h i}=P_{f c s, \max }$ ), whereas the inferior limit is determined according to the efficiency curve. As mentioned before, the efficiency of a FCS at low power is very poor due to the parasitic power. Thanks to the inferior limit, the energy management strategy avoids this unfavourable zone (Fig. 2).

This EMS gives priority to the FCS-power supply since the FCS is the primary power source and the direct power flow to the DC bus through the FCS power converter has lower losses than the indirect way through the ESS power converter, the ESS itself and again to the DC bus through the power converter to supply the load. Consequently, given the present $P_{\text {load }}(k)$, if:

$P_{f c s, l o} \eta_{B} \leq P_{\text {load }}(k) \leq P_{f c s, \max } \eta_{B}$

and

$\Delta P_{f c s, \text { fall rate }} \leq \Delta P_{\text {load }}(k) \leq \Delta P_{\text {fcs, rise rate }}$

where:

$\Delta P_{\text {load }}(k)=P_{\text {load }}(k)-P_{\text {load }}(k-1)$

then, the FCS operates in load-following mode:

$P_{f c s}(k)=P_{\text {load }}(k) / \eta_{B}$

and 


$$
P_{\text {ess }}(k)=0
$$

If, on the contrary:

$$
P_{\text {load }}(k) \leq P_{f c s, l o}(k) \eta_{B}
$$

then:

$$
P_{f c s}(k)=P_{f c s, l o}(k)
$$

and the ESS stores the rest of generated power if the ESS is not too charged:

$$
P_{e s s}(k)=-\min \left\{\left|P_{\text {load }}(k)-P_{f c s}(k) \eta_{B}\right| \eta_{\text {ess }} \eta_{B / B}, \quad\left|S o E(k)-S o E_{\max }\right| k_{e s s}\right\}
$$

On the other hand, in the case that the ESS is full charged, then $P_{f c s}(k)=0$. Conversely, if:

$$
P_{\text {load }}(k) \geq P_{f c s, h i}(k) \eta_{B}
$$

then:

$$
P_{f c s}(k)=P_{f c s, \max }
$$

and the ESS supplies the rest of load power if there is enough energy in ESS:

$$
P_{e s s}(k)=\min \left\{\frac{\left(P_{\text {load }}(k)-P_{\text {fcs }}(k) \eta_{B}\right)}{\eta_{B / B} \eta_{\text {ess }}}, \quad\left(S o E(k)-S o E_{\text {min }}\right) k_{\text {ess }}\right\} .
$$

In Fig. 3, it is represented how is determined the FCS operating point. However, the transition between operating points is limited by the maximum rates, thus, finally:

$$
P_{f c s}(k)= \begin{cases}P_{f c s}(k-1)+\Delta P_{f c s, \text { fall rate }} \Delta T, & \text { if } \Delta P_{f c s}(k) \leq \Delta P_{f c s, \text { fall rate }} \\ P_{f c s}(k-1)+\Delta P_{f c s, \text { rise rate }} \Delta T, & \text { if } \Delta P_{f c s}(k) \geq \Delta P_{f c s, \text { rise rate }}\end{cases}
$$

where $\Delta P_{f c s}=P_{f c s}(k)-P_{f c s}(k-1)$, with $P_{f c s}(k)$ as it was previously calculated in (19), (22) or (25), according to the conditions (16), (21) or (24), respectively. The power $P_{\text {ess }}(k)$ is calculated as in indicated in (23) and (26). 


\subsection{Improved strategy based on the FCS efficiency map}

The second strategy based on the FCS efficiency map is a strategy which operates the FCS preferably in its point of maximum efficiency in order to improve the hydrogen economy, although the final operating point of the FCS is determined based on the actual power demand and the state of energy of the ESS. The FCS power command is determined according to the following rules. If the load power is:

$P_{f c s, l o} \eta_{B} \leq P_{\text {load }}(k) \leq P_{f c s, h i} \eta_{B}$

and, the $S o E$ is:

$\operatorname{SoE} E_{l o} \leq \operatorname{SoE}(k) \leq \operatorname{SoE}_{h i}$

where $P_{f c s, h i}$ is:

$P_{f c s, h i}=P_{f c s, \max } \eta_{B} X_{f c s, h i}$,

and $X_{f c s, h i}$ is a fraction of the maximum FCS power; then, the FCS is operated in its point of maximum efficiency:

$P_{f c s}(k)=P_{f c s, \max e f f}$

The remaining power to achieve the load demand flows from or to the ESS according to (26) if $P_{\text {load }}(k)>P_{f c s, \text { max eff }}$ (discharging mode), or (23) if $P_{\text {load }}(k)<P_{f c s, \text { max eff }}$ (charging mode).

If the load power is:

$P_{f c s, h i} \eta_{B} \leq P_{\text {load }}(k) \leq P_{f c s, \max } \eta_{B}$

and the $S o E$ is:

$S o E_{l o} \leq \operatorname{SoE}(k) \leq S o E_{h i}$

then, the FCS is operated in load following mode:

$P_{f c S}(k)=P_{\text {load }}(k) / \eta_{B}$

and $P_{\text {ess }}(k)$ is as indicated in (23) or (26). 
On the other hand, if:

$P_{\text {load }}(k) \geq P_{f c s, \max } \eta_{B}$ and $\operatorname{SoE}(k) \leq S o E_{h i}$

or:

$\operatorname{SoE}(k) \leq \operatorname{SoE}_{l o}$

then, the FCS is operated at its maximum power:

$P_{f c s}(k)=P_{f c s, \max }$

and $P_{\text {ess }}(k)$ is as indicated in (26). If, on the contrary:

$P_{\text {load }}(k) \leq P_{f c s, l o} \eta_{B}$ and $\operatorname{SoE}(k) \geq \operatorname{SoE} E_{l o}$,

or:

$\operatorname{SoE}(k) \geq S_{o} E_{h i}$

then, the FCS is operated at its lower operating point:

$P_{f c s}(k)=P_{f c s, l o}$,

and $P_{\text {ess }}(k)$ is as in (23). Additionally, if $P_{\text {load }}(k)=0 \forall t \in[k 1, k 2]$ with $(k 2-k 1)>T_{\text {off }}$, and, $\operatorname{SoE}(k)>\operatorname{So} E_{h i}$ with $k>k 2$, then, the FCS is turned off to avoid unnecessary hydrogen consumption because the parasitic losses in the FCS. Figure 4 indicates the FCS operating point as a function of the $S o E(k)$ and the load power $P_{\text {load }}(k)$. In the same way that in the previous strategy, the transition between operating points is realized according to the constraints concerning the maximum fall power rate and the maximum power rate as indicated in (27).

\subsection{Strategy based on constrained nonlinear programming}

In this strategy, an optimization problem with linear constraints is resolved at each sampling period $\Delta T$ where a nonlinear cost function, which represents the hydrogen consumption, is minimized. The problem can be put into the form [28]: 
$\min _{X} J(x)$ subject to $H(X)=0$ and $\quad G(X) \leq 0$

where the vector $X$ is:

$X=\left[\begin{array}{c}P_{f c s} \\ P_{e s s}\end{array}\right]$.

The cost function $J(X)$ represents the hydrogen consumption in the FCS:

$J(X)=F\left(P_{f c s}(k)\right)$

where

$F\left(P_{f c s}(k)\right)=\operatorname{ConsH}_{2}\left(P_{f c s}(k)\right) P_{f c s}(k) \Delta T$

and $\operatorname{ConsH}_{2}\left(P_{f c S}(k)\right)$ is the hydrogen consumption $\left(\mathrm{g} \mathrm{Wh}^{-1}\right)$ as a function of $P_{f c S}(k)$. This relationship is shown in the form of a consumption map in Fig. 2.

The set of constraints $H(X)=0$ represents the power balance in the DC bus as in (6) and the set of constraints $G(X) \leq 0$ represents the limitations in $P_{f c s}(k)$ and $P_{e s s}(k)$. The $P_{f c s}(k)$ is limited in its maximum and minimum value, and in the maximum rise rate and fall rate, thus:

$$
\begin{aligned}
& P_{f c s, \max }(k)=\max \left[P_{f c s, h i} ; \quad P(k-1)+\Delta P_{f c s, \text { fall rate }} \Delta T\right] \\
& P_{f c s, \text { min }}(k)=\min \left[P_{f c s, l o} ; \quad P(k-1)+\Delta P_{f c s, \text { rise rate }} \Delta T\right]
\end{aligned}
$$

On the other hand, $P_{\text {ess }}(k)$ is limited to its maximum or minimum value depending on the actual $\operatorname{SoE}(k)$. The $\operatorname{SoE}(k)$ is limited:

$$
S o E_{\min } \leq \operatorname{SoE}(k) \leq S o E_{\max }
$$

thus:

$$
k_{\text {ess }}\left(\operatorname{SoE}(k)-S o E_{\max }\right) \leq P_{e s s}(k) \leq k_{\text {ess }}\left(\operatorname{SoE}(k)-S o E_{\min }\right)
$$

where $k_{\text {ess }}$ is the constant defined in (15). 


\section{Simulation results}

The enunciated strategies were tested by simulation with a model of a hybrid system consisting in the vehicle described in Table 1, provided with a FCS and a supercapacitors-based ESS with the principal parameters listed in Table 2. The supercapacitor bank is sized in order to accomplish the following acceleration requirement: the vehicle (with total mass $1380 \mathrm{~kg}$ including an extra cargo mass) must be capable to accelerate from 0 to $96.5 \mathrm{kph}$ in $10 \mathrm{~s}$ with a $15-\mathrm{kW}$ FCS. The fuel cell is sized in order to be possible to fulfil all the Standard Driving Cycles described in Section 4.1. On the other hand, the strategy parameters $P_{f c s, l o}$ and $P_{f c s, h i}$ are adjusted in order to maximize the fuel economy and performance according to the particular strategy and cycle. Their values are shown in Table 3.

\subsection{Testing the EMSs with standard driving cycles}

The strategies were tested on four Standard Driving Cycles: the New European Driving Cycle (NEDC), the Urban Dynamometer Driving Schedule (UDDS), the Federal Test Procedure (FTP), and the Highway Fuel Economy Test (HWFET). These speed profiles, representing urban and highway scenarios, were originally stated for measuring pollutant emissions and gasoline economy of engines [29] and are widely used in the literature to evaluate the performance of EMSs and to assess the hybridization degree (i.e., the parameter that indicates the relation between the maximum power of the fuel cell and the maximum power of the energy storage system).

To illustrate the behaviour of the proposed strategies, the simulation results corresponding to the NEDC cycle are shown: the power split between the fuel cell system and the energy storage system, and the evolution of the $S o E(t)$ in the ESS are 
shown in Fig. 5 for the strategy based on efficiency map, in Fig. 6 for the improved strategy based on efficiency map and in Fig. 7 for the strategy based on constrained nonlinear programming. These figures are only plotted from time 960 to $1180 \mathrm{~s}$ for clarity.

\subsection{Comparative results}

A comparative analysis of the strategies for the four cycles, in terms of hydrogen consumption per kilometre, is shown in Fig. 8. The comparative is done with respect to the optimal case where the consumption is minimum; the values in percentage indicate the increment in consumption with respect to the optimal case.

The performance of the optimal case is estimated assuming that the entirely cycle is known a priori and operating the FCS during the entirely cycle with maximum efficiency. The proposed procedure to evaluate this minimum consumption is based on the following assumptions:

- The capacity of storage in the ESS is sufficient to recover all the available energy from regenerative braking.

- The friction brake is not employed during the entire cycle.

- The power consumption, when the FCS is turned off, is null.

- The FCS is already hot when the cycle starts.

- The entire vehicle mass is fixed for all the cycles: $m_{v e h}=1109 \mathrm{~kg}$.

The procedure to determine the optimal hydrogen consumption is as follows. A simulation is performed for each driving cycle in such a way that the FCS works alternately in two operating points, namely "On" and "Off", according to the actual $\operatorname{SoE}(k)$ : i) when $\operatorname{SoE}(k)<\operatorname{SoE}(0)$, the FCS is operated at its point of maximum 
efficiency (the "On" point) and, ii) when $\operatorname{SoE}(k)>\operatorname{SoE}(0)$, the FCS is turned off (the "Off" point). As a result, the final state of energy $(\operatorname{SoE}(N))$ is the same as the initial state of energy $(\operatorname{SoE}(0))$. Thus, the hydrogen consumption only accounts the necessary hydrogen to fulfil the cycle of duration $N$. The advantages of this method are considered in the discussion Section.

In Fig. 9, it is shown a scheme with the FCS operation to perform the analysis previously described and the results are recollected in Table 4. Since the FCS is either working at the point of maximum efficiency or it is turned off (with the assumption that, when the FCS is turned off, the power consumption is null), the minimum consumption is guaranteed. In addition, in Fig. 10 is included the performance corresponding to the pure fuel cell case where there is no hybridization. This analysis is performed with a 37.5-kW FCS, a power that is sufficient to fulfil the four cycles in study.

\subsection{Influence of the initial SoE}

In general, the final state of charge $\operatorname{SoE}\left(N_{c}\right)$ is different to the initial state of charge $\operatorname{SoE}(0)$, resulting in a gain or a loss of energy in the ESS over the driving cycle. Because of that, the results are corrected in order to compare the results correctly. The corrected consumption of hydrogen is based on the assumption that after the cycle, the FCS is continuing running in its point of maximum efficiency until the $S o E$ reaches again the initial value. Thus, the corrected consumption results:

Cons $H_{2, \text { corrected }}=$ Cons $H_{2}+\frac{\Delta E_{\text {ess, stored }}}{L H V_{H 2} \eta_{B} \eta_{B / B} \eta_{e s s} \eta_{f c s, \max }}$

where, Cons $\mathrm{H}_{2}$ is the cumulative consumption of hydrogen over the cycle previous to the correction, $\Delta E_{\text {ess }}$, stored is the difference of the energy stored in the ESS at the end 
of the cycle with the energy at the beginning of the cycle (positive if $\operatorname{SoE}\left(N_{c}\right)>\operatorname{SoE}(0)$ and negative in the opposite situation), $L H V_{H^{2}}$ is the low heating value of hydrogen, $\eta_{f c s, \max }$ is the maximum efficiency of the FCS and $\eta_{e s s}$ is the ESS efficiency. In Fig. 11 and 12 , it is shown the influence of the initial $\mathrm{SoE}$ on the hydrogen consumption running on UDDS and HWFET, respectively.

\section{Experimental validation}

This section presents the implementation of the previously developed EMSs in an experimental test setup. The arrangement of this Section is as follows. First, the experimental setup is described. Then, the experimental results are presented showing the temporal behaviour, the performance in terms of hydrogen consumption, and the comparative between EMSs. Finally, the conclusions of this section are presented.

\subsection{Description of the experimental setup}

The experimental setup is a test station in which some components of the FCHV are actually present, some are emulated, and some others are simulated. The objective of this setup is to reproduce, as close as possible, the FCHV behaviour in order to validate the EMSs. In this experimental setup (shown in Fig. 13), the FCS is a NEXA power

module from Ballard of $1.2 \mathrm{~kW}$. The power module is fed with hydrogen from a pressurized tank and the hydrogen flow is measured with a mass flow meter (Bronkhorst, model F-201AC), with a maximum flow capacity of 50 SLPM.

The vehicle power consumption is emulated with a programmable electronic load (Höcherl \& Hackl, model ZS1606), a power device that can support up to $1600 \mathrm{~W} @ 60$ $\mathrm{V}, 150 \mathrm{~A}$. This load is commanded by the Host computer, which is in charge of carrying 
out the EMSs. The ESS is simulated with a model developed in the LabVIEW environment. LabVIEW is a platform for a visual programming language from National Instruments. This platform is commonly used for data acquisition, instrument control, and industrial automation. For more detail see [30].

The supercapacitor bank is composed of 130 modules and each module is composed of six serially connected cells. The main characteristics of these devices are listed in Table 5 and correspond to a module from Maxwell Technologies [31]. In this experimental stage the supercapacitor bank is scaled in the same proportion as is the fuel cell $(15 \mathrm{~kW}$ to $1.2 \mathrm{~kW}$ ). The scaled experimental supercapacitor bank is, thus, composed of 10 modules, the maximum energy that can be stored is $18.15 \mathrm{Wh}$, and the maximum power that can be delivered is $14.25 \mathrm{~kW}$. The SoE is calculated at each sampling time according to this model, which is running in the Host computer.

The data acquisition and control system is composed by a Host computer and a computer running in real time, namely RTOS computer. The RTOS computer communicates with the input/output (I/O) modules, made by National Instrument, throughout a FPGA target and a PCI bus in a second computer. The two computers are connected via an Ethernet link. The Host computer also allows monitoring the evolution of the system variables and commanding the system through a graphical interface developed in LabVIEW. The measured variables are the hydrogen flow, the load current and the stack voltage. This variables are measured every $200 \mathrm{~ms}$.

The operation of the experimental setup is as follows. Firstly, the FCS operation is determined according to the EMSs that were described in Section 3. To that end, the 
EMSs were programmed in the LabVIEW environment. These applications run in the RTOS computer and assess a new request to the FCS, $P_{f c s}(k)$, every $1 s$. Then, the $P_{f c s}(k)$ is required to the NEXA power module through the electronic load, which is commanded from the RTOS computer. The electronic load is operated in power mode, i.e., the load sinks a power in accordance with the external-programming signal from the RTOS computer. On the other hand, the $P_{\text {ess }}(k)$ value is determined from the power balance in the DC bus as in (6) and is utilized in the ESS model, running in the Host computer, to actualize the actual $\operatorname{SoE}(k)$.

\subsection{Experimental results}

The previously explained experimental setup was utilized to validate the enunciated EMSs running on the same four driving cycles in Section 4. In the simulation stage, it was utilized a $15-\mathrm{kW}$ FCS to fulfil the driving cycles. In this stage, all the powers are scaled down with a factor of 12.5 to fit with the rated power of the NEXA power module $(1.2 \mathrm{~kW})$. In Fig. 14 is shown the comparative between the experimental efficiency map of NEXA and the efficiency map of the FCS model in the ADVISOR simulator. The experimental map is obtained measuring the inlet hydrogen flow rate according to:

$\eta_{f c s}=\frac{3600}{\operatorname{Cons}_{H 2} L H V_{H 2}} \quad[\%]$,

where Cons $_{H 2}$ is in $g \mathrm{kWh}^{-1}$ and $L H V_{H 2}=120 \mathrm{~kJ} \mathrm{~g}^{-1}$. In Fig. 15, 16, and 17 are shown the experimental results corresponding to the NEDC cycle, showing the evolution of FCS power $P_{f c s}$, measuring the stack voltage and the stack current; the evolution of $S o E$, from the ESS model; and the hydrogen consumption, measured with a mass flow meter. 
In Fig. 18, it is shown the comparative of the hydrogen consumption between the strategies in the experimental setup and the strategies in the simulation environment. To do this comparative the experimental results are multiplied by the same scale factor used to scale down the power. From this comparative, it is possible to draw some observation. Firstly, the hydrogen consumption corresponding to the experimental setup is higher than the corresponding to the simulation setup. This is owing to the fact that the efficiency of the NEXA is lower than the efficiency of the FCS model in the simulation setup as can be seen in Fig. 14. Secondly, the relative consumptions in the experimental setup are similar to the experimental setup with some differences owing to discrepancies in the modelling and measurement errors.

On the other hand, in Fig. 19 is shown the hydrogen savings achieved using the EMSs with respect to the pure fuel cell case with no hybridization. The results show the interest of hybridization, achieving hydrogen savings up to $33.7 \%$.

\section{Discussion}

The analysis of the hydrogen economy in Fig. 8 shows that the three strategies have a good performance compared to the optimal case where the entire driving cycle is known a priori, which is not feasible in practice. In fact, the maximum deviation from the optimal case is $9.6 \%$, from the strategy based on efficiency map running on NEDC and the minimum deviation is $4.0 \%$ in the improve strategy based on efficiency map running on UDDS. The strategy based on constrained nonlinear programming gives the best performance in all the cases; however, the performance is similar to the two strategies based on efficiency map. On the other hand, compared to the pure fuel cell case (Fig. 10), the results show considerable hydrogen savings running on cycles 
NEDC, UDDS, and FTP. On the contrary, running on HWFET the savings are exiguous. This cycle is a highway driving cycle and one of its characteristic is that the average deceleration is significantly lower $\left(-0.22 \mathrm{~m} \mathrm{~s}^{-2}\right)$ than the corresponding to the other cycles (NEDC: $-0.79 \mathrm{~m} \mathrm{~s}^{-2}$; UDDS: $-0.58 \mathrm{~m} \mathrm{~s}^{-2}$; FTP: $-0.58 \mathrm{~m} \mathrm{~s}^{-2}$ ). These results suggest that the strategies achieve the objectives satisfactorily when a significant energy from braking is available.

It is remarkable that it is possible to meet the load power in the four driving cycles with a $15-\mathrm{kW}$ fuel cell, what is significantly lower to the $37.5-\mathrm{kW}$ fuel cell that is necessary if no hybridization is present. This advantage would be translated in a reduction in the production costs and hydrogen consumption.

The analysis of the influence of the initial $S o E$ on the hydrogen consumption (Fig. 11 and 12) shows that the first strategy and the third strategy have a minimal dependence on this parameter, which denotes robustness of the strategies. On the other hand, the performance of the second strategy is dependent on the initial $S o E$, especially in the HWFET cycle. In this case, the performance is worse than in the first strategy if the initial $S o E$ is lower than $60 \%$.

The supercapacitor bank is design in order to fulfill an acceleration requirement as mentioned in Section 4. However, it is useful to analyze how the strategies work with different sizes of supercapacitor bank. When the number of supercapacitors is increased, the hydrogen consumption slightly increases because of the increment in the vehicle mass is the preponderant factor. On the contrary, when the number of supercapacitors 
starts to be decreased, the hydrogen consumption also decreases because of the mass reduction although the acceleration test is not fulfilled.

Concerning to the analysis of the optimal hydrogen consumption, in some works, e.g., $[19,32-33]$, it is performed through the use of the Dynamic Programming (DP) technique, a procedure that allows to determine a global optimal operation of the system for a given load profile by evaluating all possible control sequences in a systematic way. However, a disadvantage of this technique is the relative long computation time due to the large required grid density. The grid is the result of the discretization of time and the state variables in appropriate grid-levels. Thus, in order to obtain accurate results a high density grid has to be considered. On the contrary, in our work, this issue is resolved without discretization in the state variables, reducing the computation time without losing accuracy.

\section{Conclusions}

In this work, Energy Management Strategies for Fuel Cell Hybrid Vehicles were addressed. The objectives of the EMSs were enunciated and three strategies were proposed. These strategies are based on the knowledge of the efficiency map. The first strategy is a quasi-load-following strategy in which the FCS is operated in an advantageous zone where the efficiency is high whereas the second strategy operates the FCS preferably in its point of maximum efficiency in order to improve the hydrogen economy. In the third strategy, an optimization problem with linear constraints is resolved at each sampling period where a nonlinear cost function, which represents the hydrogen consumption, is minimized. 
Firstly, the EMSs were evaluated in a simulation environment using four standard driving cycles and, then, the EMSs were tested in an experimental setup reproducing the FCVH behaviour were some components are actually present, some are emulated and some others are simulated.

The results show that using the proposed EMSs it is possible to achieve a high reduction in the hydrogen consumption in the range of $17 \%$ average. This is achieved operating the FCS properly and taking advantage of the energy from regenerative braking. To evaluate the savings, the hydrogen consumptions are compared with two references: the pure fuel cell case with no hybridization and the optimal case with minimum consumption where the driving cycle is known a priori.

\section{Acknowledgment}

This work has been funded partially by the project CICYT DPI2007-62966 of the Spanish Government and the Department of Universities, Investigation and Society of Information of the Generalitat de Catalunya. We counted with the support of Fuel Cells Laboratory of the Institute of Robotics and Industrial Informatics (IRI).

\section{References}

[1] P. Zegers, Journal of Power Sources, 154 (2006) 497-502.

[2] International Energy Agency, "Key World Energy Statics" http:// www.iea.org/. Last accessed 10/15/07.

[3] K. Rajashekara, in: Fuel Cell Technology for Vehicles, 2000, pp. 179-187.

[4] K. Jeong and B. Oh, Journal of Power Sources 1005 (2002) 58-65.

[5] D. Friedman and R. Moore, Proceedings Electrochemical Society 27 (1998) 407423.

[6] C. Chan, Proceedings of the IEEE, 90 (2002) 247-275.

[7] R. Helmolt and U. Eberle, Journal of Power Sources 165 (2007) 833-843.

[8] P. Thounthong, S. Raël, and B. Davat, Journal of Power Sources 158(2006) 806814.

[9] J. Jung, Y. Lee, J. Loo, and H. Kim, in: Fuel Cell for Transportation, 2003, pp. 
201-205.

[10] G. Paganelli, Y. Guezennec, and G. Rizzoni, Optimizing Control Strategy for Hybrid Fuel Cell Vehicle, 2002, pp. 71-79.

[11] P. Rodatz, O. Garcia, L. Guzzella, F. Büchi, M. Bärschi, T. Tsukada, P. Dietrich, R. Kotz, G. Schreder, and A.Woukan, in: Fuel Cell Power for Transportation, 2003, pp. 77-84.

[12] P. Rodatz, G. Paganelli, A. Sciarretta, and L. Guzzella, Control Engineering Practice, 13 (2004) 41-53.

[13] E. Karden, S. Plounen, B. Fricke, T. Miller, and K. Snyder, Journal of Power Sources, 168 (2007) 2-11.

[14] R. Kötz, S. Müller, M. Bärtschi, B. Schnyder, P. Dietrich, FN Büchi, A. Tsukada, GG Scherer, P. Rodatz, O. Garcia, ElectroChemical Society (2001) 2-7.

[15] S. Caux, J. Lachaize, M. Fadel, P. Shott, and L. Nicod, Journal of Process Control, 15 (2005) 481-491.

[16] S. Caux, J. Lachaize, M. Fadel, P. Shott, and L. Nicod, In Proceedings of the 16th IFAC World Congress, Prague, 2005.

[17] J. Schiffer, O. Bohlen, RW de Doncker, and DU Sauer, Vehicle Power and Propulsion, 2005 IEEE Conference, (2005) 716-723.

[18] M. Kim and H. Peng, Journal of Power Sources, 165 (2007) 819-832.

[19] J. Kessels. Energy Management for Automotive Power Nets. PhD thesis, Technische Universiteit Eindhoven, 2007.

[20] T. Markel, A. Brooker, T. Hendricks, V. Johnson, K. Kelly, B. Kramer, M. O'Keefe, S. Sprik, and K. Wipke, Journal of Power Sources, 110 (2002) 255266.

[21] K. Wipke, M. Cuddy, and S. Burch, IEEE Transactions on Vehicular Technology, 48 (1999) 1751-1761.

[22] J. Pukrushpan. Modelling and Control of Fuel Cell Systems and Fuel Processors. $\mathrm{PhD}$ thesis, University of Michigan, 2003.

[23] K. Williams, W. Keith, M. Marcel, T. Haskew, Shepard, and B. Todd, Journal of Power Sources, 163 (2006) 971-985.

[24] R. Ahluwalia and X. Wang, Journal of Power Sources, 139 (2005) 152-164.

[25] J. Pukrushpan, A. Stefanopoulou, and H. Peng, IEEE Control Systems Magazine, 24 (2004) 30-46.

[26] F. Philipps, G. Simons, and K. Schiefer, Journal of Power Sources, 154 (2006) 412-419.

[27] D. Feroldi and M. Serra and J. Riera, Journal of Power Sources, 169 (2007) 205212

[28] P. Gill, W. Murray, and M. Wright, Practical optimization, London Academic Press, 1981.

[29] DieselNet, "Emission test cycles" http:// www.dieselnet.com/standards/cycles/. Last accessed 04/06/08.

[30] National Instrument. "Introducing LabVIEW 8.6" http://www.ni.com/labview/. Last accessed 23/07/08.

[31] Mawwell Technologies, "Electrical Double Layer Capacitor: Boostcap Ultracapacitor Series: BPAK" http://www.maxwell.com/ ultracapacitors/ index.asp. Last accessed 03/20/2006.

[32] M. Koot, A. Kessels, B. de Jager, W. Heemels, P. van den Bosch, and M. Steinbuch, IEEE Transaction on Vehicular Technology, 54 (2005) 771-782.

[33] T. Hofman, M. Steinbuch, R.M. van Druten, and A.F.A. Serrarens, In Proceedings of the 17th IFAC World Congress, (2008) 5652-5657. 


\section{Nomenclature}

$\Delta P_{f c s, \text { fall rate }}$ Maximum fall rate of $P_{f c s}$

$\Delta P_{f c s, \text { rise rate }} \quad$ Maximum rise rate of $P_{f c s}$

$\Delta T \quad$ Sampling time

$\eta_{B} \quad$ Efficiency of the Boost converter

$\eta_{B / B} \quad$ Efficiency of the Buck/Boost converter

$\eta_{\text {ess }} \quad$ Efficiency of the ESS

$\eta_{f c s, \max } \quad$ Maximum efficiency of the FCS

Cons $_{\mathrm{H} 2} \quad$ Hydrogen consumption

$C_{R} \quad$ Capacitance of the supercapacitors

DC Direct Current

$E_{\text {cap }} \quad$ Maximum energy that the ESS is capable to store

EMS Energy Management Strategy

ESS Energy Storage System

FC Fuel Cell

FCS Fuel Cell System

FCHV Fuel Cell Hybrid Vehicle

$k_{\text {ess }} \quad$ Constant of the ESS

$L H V_{H 2} \quad$ Low Heating Value of hydrogen

M Mass

$N_{c} \quad$ Number of sampling time in a driving cycle

$P \quad$ Power

$P_{f c s, h i} \quad$ Parameter in the EMSs

$P_{f c s, \text { low }} \quad$ Parameter in the EMSs 


$\begin{array}{ll}P_{f c S, \max } & \text { Rated fuel cell power } \\ R_{d} & \text { Internal resistance of the supercapacitors } \\ \text { SoE } & \text { State of Energy in the ESS } \\ t_{c} & \text { Duration of a driving cycle }\end{array}$

\section{Captions of the figures}

Fig. 1. FCHV scheme

Fig. 2. Efficiency and hydrogen consumption map for a 50-kW FCS as a function of the power.

Fig. 3. FCS operating point for the strategy based on efficiency map.

Fig. 4. FCS operating point for the improved strategy based on efficiency map.

Fig. 5. Power split running on NEDC and the evolution of the SoE using the strategy based on efficiency map.

Fig. 6. Power split running on NEDC and the evolution of the SoE using the improved strategy based on efficiency map.

Fig. 7. Power split running on NEDC and the evolution of the SoE using the strategy based on constrained nonlinear programming.

Fig. 8. Comparative between the hydrogen consumption of the proposed strategies, the optimum case and the pure fuel cell case. The percentages in red indicate the increment in consumption with respect to the optimal case.

Fig. 9. Scheme showing the On/Off operation of the FCS model to perform the analysis of the minimum hydrogen consumption.

Fig. 10. Hydrogen savings with respect to the pure fuel cell case.

Fig. 11. Influence of the initial SoE on the hydrogen consumption running on UDDS.

Fig. 12. Influence of the initial SoE on the hydrogen consumption running on HWFET. 
Fig. 13. Scheme showing the experimental setup to validate the EMSs.

Fig. 14. Comparative between the experimental efficiency map of NEXA and the efficiency map of ADVISOR model.

Fig. 15. Experimental results of the strategy based on efficiency map.

Fig. 16. Experimental results of the improved strategy based on efficiency map.

Fig. 17. Experimental results of the strategy based on constrained nonlinear programming.

Fig. 18. Experimental validation of the proposed strategies.

Fig. 19. Hydrogen savings achieved using the EMSs with respect to the pure fuel cell case. 